\title{
Evakuierungen durch Überschwemmungen erfordern psychische Versorgung Betroffener
}

\begin{abstract}
Ob Hurrikane wie Harvey oder Irma in den USA oder die aktuellen Überschwemmungen durch den Monsun in Indien - bei den betroffenen Menschen hinterlassen Naturkatastrophen nicht nur materielle Schäden. Viele leiden auch psychisch unter Depressionen oder Posttraumatischen Belastungsstörungen. Aber was haben Pflegekräfte damit zu tun?
\end{abstract}

» Überschwemmungen sind nicht nur mit dem Verlust von Eigentum und physischen Verletzungen verbunden, sondern auch mit psychischem Stress für die Betroffenen. Eine nationale Studie von A. Munro et al. untersuchte dies nach dem Hochwasser in England im Jahr 2013/2014 genauer.

In der Querschnittsstudie wurden dafür 622 Personen ab 18 Jahren analysiert, deren Häuser von der Überschwemmung betroffen waren. Von ihnen hatten $72 \%$ (449) ihre Häuser verlassen müssen. Die drei primären Outcomes wurden anhand validierter Instrumente gemessen und Score-Werte für die Einstufung festgelegt:

- Depression: Patient-Health Questionnaire Depression Scale (PHQ-2), Score $\geq 3$

- Angst: Generalised Anxiety Disorder Anxiety Scale (GAD-2), Score $\geq 3$

- Posttraumatische Belastungsstörung: Post-Traumatic Stress Disorder Checklist (PCL-6), Score $\geq 14$
Es zeigte sich, dass Personen, die evakuiert wurden, ein Jahr nach der Überschwemmung häufiger Symptome von Depression, Angst und Posttraumatischer Belastungsstörung aufwiesen, im Vergleich zu Personen, deren Häuser zwar von dem Hochwasser betroffen waren, aber nicht evakuiert werden mussten. Mittels einer Regressionsanalyse konnte weiterhin ermittelt werden, dass diejenigen, die vor der Überschwemmung und Evakuierung keine Warnung erhalten hatten, signifikant häufiger Symptome einer Depression und einer Posttraumatischen Belastungsstörung aufwiesen, jedoch keine Symptome von Angst. Eine rechtzeitige Warnung (mindestens zwölf Stunden vorher) stellte sich als einziger Determinant heraus, der protektiv auf die Entstehung von psychischen Störungen bei evakuierten Menschen wirkte.
Quelle

Munro A, Kovats RS, Rubin GJ, Waite TD, Bone A, Armstrong B, and the English National Study of Flooding and Health Study Group. Effect of evacuation and displacement on the association between flooding and mental health outcomes: a cross-sectional analysis of UK survey data. The Lancet Planetary Health 2017; 1(4): e134-e141. DOI: 10.1016/S25425196(17)30047-5

\section{Kommentar}

Durch die Studie wurden die langfristigen psychischen Auswirkungen in Folge von Hochwasser und Überschwemmungen in besiedelten Gebieten epidemiologisch beschrieben. Die Ergebnisse zeigten, dass nach Evakuierungen vor allem auch eine psychische Versorgung der Betroffenen notwendig wird. Fraglich bleibt, ob all die von psychischen Folgen betroffenen Personen eine entsprechende professionelle Behandlung aufsuchten, um Langzeitfolgen, die sich auch auf den körperlichen Bereich auswirken können, zu vermindern oder sogar zu verhindern. Damit beispielsweise Auswirkungen mit Krankheitswert frühzeitig erkannt werden, wäre ein systematisches Screening von Betroffenen nach Naturereignissen notwendig. In Deutschland fehlen dazu jedoch entsprechende Strukturen, trotz regelmäßig auftretender Hochwasser und Überschwemmungen. Aufgrund ihrer fachlichen Qualifikation wären Pflegekräfte eine Berufsgruppe, die bei der frühzeitigen Erkennung von hochwasserbedingten psychischen Problemen eingebunden werden könnte. Daher wäre zu überlegen, wie Pflegekräfte sensibilisiert und qualifiziert werden können, psychische Probleme bei Personen, die von den Auswirkungen einer Überschwemmung betroffen sind, zu erkennen, um deren psychische Gesundheit zu erhalten. Die Untersuchung von sozioökonomischen Merkmalen der Betroffenen könnte ferner dazu beitragen, die Personen zu identifizieren, welche besonders gefährdet sind eine psychische Symptomatik nach einer solchen Großschadenslage zu entwickeln, um bei der Nachsorge zielgerichtet vorgehen zu können.

\section{Autorenkontakt:}

Referiert und kommentiert von

Michéle Müller, Master of Science Gesundheitswissenschaften, Zwickau E-Mail: michele1992-Mueller@ web.de 